\title{
Chemical potential of a test hard sphere of variable size in a hard-sphere fluid
}

\author{
David M. Heyes ${ }^{1, a)}$ and Andrés Santos ${ }^{2}, \mathrm{~b}$ ) \\ ${ }^{1)}$ Department of Physics, University of London, Egham, Surrey TW20 OEX, \\ $U K$ \\ ${ }^{2)}$ Departamento de Física and Instituto de Computación Científica Avanzada (ICCAEx), Universidad de Extremadura, \\ E-06071 Badajoz, Spain
}

(Dated: 16 October 2018)

The Labík and Smith Monte Carlo simulation technique to implement the Widom particle insertion method is applied using Molecular Dynamics (MD) instead to calculate numerically the insertion probability, $P_{0}\left(\eta, \sigma_{0}\right)$, of tracer hard-sphere (HS) particles of different diameters, $\sigma_{0}$, in a host HS fluid of diameter $\sigma$ and packing fraction, $\eta$, up to 0.5 . It is shown analytically that the only polynomial representation of $-\ln P_{0}\left(\eta, \sigma_{0}\right)$ consistent with the limits $\sigma_{0} \rightarrow 0$ and $\sigma_{0} \rightarrow \infty$ has necessarily a cubic form, $c_{0}(\eta)+c_{1}(\eta) \sigma_{0} / \sigma+c_{2}(\eta)\left(\sigma_{0} / \sigma\right)^{2}+$ $c_{3}(\eta)\left(\sigma_{0} / \sigma\right)^{3}$. Our MD data for $-\ln P_{0}\left(\eta, \sigma_{0}\right)$ are fitted to such a cubic polynomial and the functions $c_{0}(\eta)$ and $c_{1}(\eta)$ are found to be statistically indistinguishable from their exact solution forms. Similarly, $c_{2}(\eta)$ and $c_{3}(\eta)$ agree very well with the Boublík-Mansoori-Carnahan-Starling-Leland and Boublík-CarnahanStarling-Kolafa formulas. The cubic polynomial is extrapolated (high density) or interpolated (low density) to obtain the chemical potential of the host fluid, or $\sigma_{0} \rightarrow \sigma$, as $\beta \mu^{\text {ex }}=c_{0}+c_{1}+c_{2}+c_{3}$. Excellent agreement between the Carnahan-Starling and Carnahan-Starling-Kolafa theories with our MD data is evident.

\section{INTRODUCTION}

The statistical mechanical theory of hard-sphere (HS) fluids and solids is important as it underpins the phase behavior and physical properties of a wide range of condensed phase systems such as simple liquids, glasses, colloidal particles, emulsion droplets, and granular materials. ${ }^{1}$ This work reports Molecular Dynamics (MD) simulations to test accurate analytic expressions for the chemical potential of a HS impurity of variable diameter at infinite dilution in a HS fluid. This information is a useful precursor for understanding tracer solubility and HS mixtures in general.

We consider a test (or impurity) HS of diameter $\sigma_{0}$ immersed in a sea of HSs of diameter $\sigma$ at a packing fraction $\eta .^{2}$ The quantity of interest here is the excess chemical potential of the test particle, $\mu_{0}^{\mathrm{ex}}\left(\eta, \sigma_{0}\right)$, which becomes identical to the excess chemical potential $\mu^{\operatorname{ex}}(\eta)$ of the host fluid in the limit $\sigma_{0} \rightarrow$ $\sigma$, i.e., $\lim _{\sigma_{0} \rightarrow \sigma} \mu_{0}^{\mathrm{ex}}\left(\eta, \sigma_{0}\right)=\mu^{\mathrm{ex}}(\eta)$. As proved by Widom, ${ }^{3-5}$ the probability $P_{0}\left(\eta, \sigma_{0}\right)$ of successful insertion of the test particle is related to the chemical potential through

$$
P_{0}\left(\eta, \sigma_{0}\right)=e^{-\beta \mu_{0}^{\mathrm{ex}}\left(\eta, \sigma_{0}\right)}
$$

where $\beta=1 / k_{B} T$ and $k_{B}$ is Boltzmann's constant.

The particle insertion technique has been applied to HS fluids for many decades..$^{2,3,6-11}$ However, if $\eta$ is rather large and $\sigma_{0}=\sigma$, the insertion probability is so small that the method becomes inefficient to measure directly

\footnotetext{
a)Electronic mail: david.heyes@rhul.ac.uk b) Electronic mail: http://www.unex.es/eweb/fisteor/andres/
}

$\mu^{\mathrm{ex}}(\eta)$ in computer simulations. In those situations, a circumventing path is needed.

Labík and Smith (LS) ${ }^{9}$ proposed a NVT Monte Carlo (MC) simulation technique which can achieve this $\sigma_{0} \rightarrow$ $\sigma$ limit accurately even at high densities. The method measures the probability of the successful insertion of a solute particle with a range of diameter values, $\sigma_{0}$, smaller than that of the solvent HS diameter. These measurements are extrapolated with a suitable polynomial in powers of $\sigma_{0}$ to $\sigma_{0} \rightarrow \sigma$, giving the chemical potential of the HS solvent. Inter alia they give the tracer chemical potential of the test HS particle of diameter $\sigma_{0}<\sigma$. The technique was subsequently extended to fused HS diatomics ${ }^{10}$ and HS mixtures. ${ }^{11}$

We note that recently Baranau and Tallarek $(\mathrm{BT})^{2}$ applied a solution consisting of measuring the so-called pore-size distribution, fitting it to a Gaussian, and then performing analytically the integral in their Eq. (11) to finally determine the chemical potential. This is an alternative route to the chemical potential of the test particle in the $\sigma_{0} \rightarrow \sigma$ limit.

In this work we follow instead the LS method to calculate numerically the insertion probability, $P_{0}\left(\eta, \sigma_{0}\right)$, for different tracer $\mathrm{HS}$ sizes $\sigma_{0}$, in a host HS fluid simulated by MD. The simulation obtained $-\ln P_{0}\left(\eta, \sigma_{0}\right)$ values are fitted to a cubic polynomial $c_{0}(\eta)+c_{1}(\eta) \sigma_{0} / \sigma+c_{2}(\eta)\left(\sigma_{0} / \sigma\right)^{2}+c_{3}(\eta)\left(\sigma_{0} / \sigma\right)^{3}$ (a test function supported by several approximations), and then this polynomial is used to extrapolate (high density) or interpolate (low density) to the value of this quantity at the desired diameter $\sigma$. As mentioned above, a bonus from this way is that we obtain the chemical potential $\mu_{0}\left(\eta, \sigma_{0}\right)$ for a tracer particle with a diameter both smaller and (for some densities) also larger than $\sigma$ (not only for a fluid particle of the same size as the host fluid HSs). The density-dependent coefficients $c_{n}$ are also determined, which enables a more detailed comparison with theoretical predictions to be made. Instead of 
comparing only the chemical potential of the host fluid particle (i.e., $c_{0}+c_{1}+c_{2}+c_{3}$ ) as a function of density (as was done, for instance, in Fig. 1(a) of BT's paper), we validate the accuracy of the simulations by (i) confirming agreement with the exact $c_{0}$ and $c_{1}$ and (ii) comparing two extra coefficients $\left(c_{2}\right.$ and $\left.c_{3}\right)$ with literature theoretical predictions, which builds on the pioneering LS work. ${ }^{9}$

The remainder of this paper is organized as follows. The standard theoretical approximations are reviewed in Sec. II and the use of a cubic polynomial as a trial function for $\beta \mu_{0}^{\mathrm{ex}}$ is justified. Section III summarizes the Widom particle insertion method and describes the way it is implemented in our MD simulations. The results are presented and compared with theoretical predictions in Sec. IV. Finally, the paper is closed with some conclusions in Sec. V.

\section{THEORETICAL APPROXIMATIONS}

\section{A. Multi-component hard-sphere fluids}

Let us start by considering a (three-dimensional) fluid mixture of additive HSs with an arbitrary number of components. There are $N_{j}$ spheres of species $j$ having a diameter $\sigma_{j}$, so that the total number of particles is $N=\sum_{j} N_{j}$ and the $n$th moment of the size distribution is

$$
M_{n}=\frac{\sum_{j} N_{j} \sigma_{j}^{n}}{N} .
$$

The total packing fraction is

$$
\eta=\frac{\pi}{6} \frac{N}{V} M_{3}
$$

where $V$ is the volume of the system.

We will denote the compressibility factor of the mixture by $Z\left(\eta,\left\{N_{j}\right\}\right) \equiv p V / N k_{B} T$, where $p$ is the pressure. Since its exact form is not known, several approximations have been proposed. ${ }^{12,13}$ In particular, the exact solution ${ }^{14-16}$ of the Percus-Yevick (PY) integral equation ${ }^{17}$ allows one to obtain explicit expressions for $Z\left(\eta,\left\{N_{j}\right\}\right)$ through different thermodynamic routes. The virial (PY-v), compressibility (PY-c), and chemicalpotential $(\mathrm{PY}-\mu)$ routes in the PY approximation share the following common structure: ${ }^{14-16,18-20}$

$$
Z\left(\eta,\left\{N_{j}\right\}\right)=Z_{0}(\eta)+Z_{1}(\eta) \frac{M_{1} M_{2}}{M_{3}}+Z_{2}(\eta) \frac{M_{2}^{3}}{M_{3}^{2}},
$$

where

$$
Z_{0}(\eta)=\frac{1}{1-\eta}, \quad Z_{1}(\eta)=\frac{3 \eta}{(1-\eta)^{2}} .
$$

The coefficient $Z_{2}(\eta)$ depends on the route and several literature predictions are displayed in Table I. On the other hand, the coefficients (2.4) are the same in all the PY approximations. As will be discussed later (see also the Appendix), those coefficients are exact.

Since none of the three prescriptions (PY-v, PY-c, and PY- $\mu$ ) is particularly accurate, Boublík ${ }^{21}$ and, independently, Mansoori et al. ${ }^{22}$ proposed an interpolation between PY-v and PY-c with respective weights $1 / 3$ and $2 / 3$. The resulting Boubliík-Mansoori-CarnahanStarling-Leland (BMCSL) compressibility factor has of course the structure (2.3) with $Z_{0}$ and $Z_{1}$ given by Eq. (2.4) and the corresponding expression for $Z_{2}$ is also included in Table I. In the monodisperse case (i.e., $\sigma_{j} \rightarrow$ $\sigma \Rightarrow M_{n} \rightarrow \sigma^{n}$ ) one has $Z=Z_{0}+Z_{1}+Z_{2}$, and the BMCSL equation of state reduces to the Carnahan-Starling (CS) one, ${ }^{18,23,24}$

$$
Z_{\mathrm{CS}}(\eta)=\frac{1+\eta+\eta^{2}-\eta^{3}}{(1-\eta)^{3}}
$$

In 1986, Kolafa proposed a slight correction to the CS equation, namely

$$
Z_{\mathrm{CSK}}(\eta)=\frac{1+\eta+\eta^{2}-\frac{2}{3} \eta^{3}(1+\eta)}{(1-\eta)^{3}}
$$

It first appeared as Eq. (4.46) in a review paper by Boublík and Nezbeda. ${ }^{25}$ Following Kolafa's recommendation ${ }^{26}{ }^{2}$ we will refer to Eq. (2.6) as the Carnahan-Starling-Kolafa (CSK) equation of state. The extension of $Z_{\mathrm{CSK}}$ to mixtures was carried out by Boublík ${ }^{27}$ by keeping the structure (2.3) and choosing $Z_{2}$ as $Z_{2}=Z_{\mathrm{CSK}}-Z_{0}-Z_{1}$. The resulting BoublíkCarnahan-Starling-Kolafa (BCSK) expression is given in the bottom row of Table I.

The excess free energy per particle of the mixture, $a^{\mathrm{ex}}\left(\eta,\left\{N_{j}\right\}\right)$, is related to the compressibility factor $Z\left(\eta,\left\{N_{j}\right\}\right)$ through $^{18}$

$$
\beta a^{\mathrm{ex}}\left(\eta,\left\{N_{j}\right\}\right)=\int_{0}^{1} d t \frac{Z\left(\eta t,\left\{N_{j}\right\}\right)-1}{t} .
$$

Therefore, the class of approximations of the form (2.3) yield

$$
\beta a^{\mathrm{ex}}\left(\eta,\left\{N_{j}\right\}\right)=c_{0}(\eta)+c_{1}(\eta) \frac{M_{1} M_{2}}{M_{3}}+a_{2}(\eta) \frac{M_{2}^{3}}{M_{3}^{2}},
$$

where

$$
\begin{gathered}
Z_{0}(\eta)=1+\eta c_{0}^{\prime}(\eta) \Rightarrow c_{0}(\eta)=-\ln (1-\eta), \\
Z_{1}(\eta)=\eta c_{1}^{\prime}(\eta) \Rightarrow c_{1}(\eta)=\frac{3 \eta}{1-\eta},
\end{gathered}
$$

$$
Z_{2}(\eta)=\eta a_{2}^{\prime}(\eta) \Rightarrow a_{2}(\eta)=\int_{0}^{1} d t \frac{Z_{2}(\eta t)}{t},
$$

the primes denoting derivatives with respect to $\eta$. The expressions for the coefficient $a_{2}(\eta)$ corresponding to the 
TABLE I. Expressions of $Z_{2}(\eta)$ [see Eq. (2.3)] and $a_{2}(\eta)$ [see Eq. (2.8)] according to several approximations.

\begin{tabular}{ccc}
\hline \hline Approx. & $Z_{2}(\eta)$ & $a_{2}(\eta)$ \\
\hline PY-v & $\frac{3 \eta^{2}}{(1-\eta)^{2}}$ & $3 \ln (1-\eta)+\frac{3 \eta}{1-\eta}$ \\
PY-c & $\frac{3 \eta^{2}}{(1-\eta)^{3}}$ & $\frac{3 \eta^{2}}{2(1-\eta)^{2}}$ \\
PY- $\mu$ & $-\frac{9 \ln (1-\eta)}{\eta}-9 \frac{1-\frac{3}{2} \eta}{(1-\eta)^{2}}$ & $\frac{9 \ln (1-\eta)}{\eta}+9 \frac{1-\frac{1}{2} \eta}{1-\eta}$ \\
BMCSL & $\frac{\eta^{2}(3-\eta)}{(1-\eta)^{3}}$ & $\ln (1-\eta)+\frac{\eta}{(1-\eta)^{2}}$ \\
BCSK & $\frac{\eta^{2}\left[3-\frac{2}{3} \eta(1+\eta)\right]}{(1-\eta)^{3}}$ & $\frac{8}{3} \ln (1-\eta)$ \\
& & $+\eta \frac{16-15 \eta+4 \eta^{2}}{6(1-\eta)^{2}}$ \\
\hline \hline
\end{tabular}

approximations PY-v, PY-c, PY- $\mu$, BMCSL, and BCSK are also included in Table I.

We now consider the excess chemical potential of a generic species $i$, which is thermodynamically defined $\mathrm{as}^{18}$

$$
\mu_{i}^{\mathrm{ex}}=\left(\frac{\partial N a^{\mathrm{ex}}}{\partial N_{i}}\right)_{V, N_{j \neq i}}
$$

In order to take the derivative in Eq. (2.8), we need to make use of the mathematical properties

$$
\begin{aligned}
& N\left(\frac{\partial \eta}{\partial N_{i}}\right)_{V, N_{j \neq i}}=\eta \frac{\sigma_{i}^{3}}{M_{3}}, \\
& N\left(\frac{\partial N M_{1} M_{2} / M_{3}}{\partial N_{i}}\right)_{V, N_{j \neq i}}= \frac{M_{1} M_{2}}{M_{3}}\left(\frac{\sigma_{i}}{M_{1}}+\frac{\sigma_{i}^{2}}{M_{2}}\right. \\
&\left.-\frac{\sigma_{i}^{3}}{M_{3}}\right), \\
& N\left(\frac{\partial N M_{2}^{3} / M_{3}^{2}}{\partial N_{i}}\right)_{V, N_{j \neq i}}=\frac{M_{2}^{3}}{M_{3}^{2}}\left(3 \frac{\sigma_{i}^{2}}{M_{2}}-2 \frac{\sigma_{i}^{3}}{M_{3}}\right) .
\end{aligned}
$$

Therefore, the final result stemming from Eq. (2.8) is

$$
\begin{aligned}
\beta \mu_{i}^{\mathrm{ex}}\left(\eta,\left\{N_{j}\right\}\right)= & c_{0}(\eta)+c_{1}(\eta) \frac{M_{1} M_{2}}{M_{3}} \frac{\sigma_{i}}{M_{1}} \\
& +\left[c_{1}(\eta) \frac{M_{1} M_{2}}{M_{3}}+3 a_{2}(\eta) \frac{M_{2}^{3}}{M_{3}^{2}}\right] \frac{\sigma_{i}^{2}}{M_{2}} \\
& +\left\{\eta c_{0}^{\prime}(\eta)+\left[\eta c_{1}^{\prime}(\eta)-c_{1}(\eta)\right] \frac{M_{1} M_{2}}{M_{3}}\right. \\
& \left.+\left[\eta a_{2}^{\prime}(\eta)-2 a_{2}(\eta)\right] \frac{M_{2}^{3}}{M_{3}^{2}}\right\} \frac{\sigma_{i}^{3}}{M_{3}} .
\end{aligned}
$$

Note that Eqs. (2.3), (2.8), and (2.12) are consistent with the exact thermodynamic relation

$$
\frac{1}{N} \sum_{i} N_{i} \beta \mu_{i}^{\mathrm{ex}}=\beta a^{\mathrm{ex}}+Z-1,
$$

thanks to the properties in (2.9), regardless of the expression for $a_{2}(\eta)$.

As proved in the Appendix (where a general dimensionality $d$ is considered), Eq. (2.12) is exact to first order in $\sigma_{i}$, i.e.,

$$
\beta \mu_{i}^{\mathrm{ex}}\left(\eta,\left\{N_{j}\right\}\right)=c_{0}(\eta)+c_{1}(\eta) \frac{M_{1} M_{2}}{M_{3}} \frac{\sigma_{i}}{M_{1}}+\mathcal{O}\left(\sigma_{i}^{2}\right) .
$$

This in turn proves the exact character of the coefficients $c_{0}$ and $c_{1}$ in Eqs. (2.9a) and (2.9b), respectively, and, hence, of the coefficients $Z_{0}$ and $Z_{1}$ in Eq. (2.4), as anticipated before.

\section{B. Test particle in a one-component hard-sphere fluid}

In this special case, we can set $M_{n} \rightarrow \sigma^{n}$ and particularize Eq. (2.12) to a species $i=0$ made of a single particle of diameter $\sigma_{0}$. The result is

$$
\beta \mu_{0}^{\mathrm{ex}}\left(\eta, \sigma_{0}\right)=c_{0}(\eta)+c_{1}(\eta) \frac{\sigma_{0}}{\sigma}+c_{2}(\eta) \frac{\sigma_{0}^{2}}{\sigma^{2}}+c_{3}(\eta) \frac{\sigma_{0}^{3}}{\sigma^{3}}
$$

where

$$
\begin{gathered}
c_{2}(\eta)=c_{1}(\eta)+3 a_{2}(\eta) \\
c_{3}(\eta)=\eta c_{0}^{\prime}(\eta)+\eta c_{1}^{\prime}(\eta)-c_{1}(\eta)+\eta a_{2}^{\prime}(\eta)-2 a_{2}(\eta) .
\end{gathered}
$$

Notice that from Eqs. (2.9) and (2.16) one can obtain the simple relation ${ }^{9}$

$$
c_{3}(\eta)=Z(\eta)-1-\frac{1}{3} c_{1}(\eta)-\frac{2}{3} c_{2}(\eta) .
$$

Inserting Eqs. (2.9a) and (2.9b) together with the approximate expressions of $a_{2}$ listed in Table I into Eqs. (2.16), one can obtain the approximate expressions for the coefficients $c_{2}$ and $c_{3}$ given in Table II. The last column of Table II presents formulas for the excess chemical potential of the fluid, i.e., $\beta \mu^{\mathrm{ex}}(\eta)=$ $\lim _{\sigma_{0} \rightarrow 1} \beta \mu_{0}^{\mathrm{ex}}\left(\eta, \sigma_{0}\right)=c_{0}(\eta)+c_{1}(\eta)+c_{2}(\eta)+c_{3}(\eta)$, for the various approximations.

Given that a number of approximations (PY-v, PYc, PY- $\mu$, BMCSL, and BCSK) share the common cubic polynomial form (2.15) (with the exact coefficients $c_{0}$ and $c_{1}$ ) for the excess chemical potential of a test particle immersed in a monodisperse HS fluid, one might reasonably query whether one could construct either a simpler approximation (with adjustable $c_{2}$ ) from a quadratic polynomial or a more accurate approximation (with adjustable $\left.c_{2}, c_{3}, c_{4}, \ldots\right)$ from a polynomial of degree higher than three. However, as we will see, if $\beta \mu_{0}^{\mathrm{ex}}\left(\eta, \sigma_{0}\right)$ is represented by a polynomial in the diameter $\sigma_{0}$, the polynomial must necessarily be of third degree. This is a consequence of the physical requirement that, in the limit of an infinitely large impurity, one must have ${ }^{28-30}$

$$
\eta Z(\eta)=\lim _{\sigma_{0} \rightarrow \infty} \frac{\beta \mu_{0}^{\mathrm{ex}}\left(\eta, \sigma_{0}\right)}{\left(\sigma_{0} / \sigma\right)^{3}} .
$$


TABLE II. Expressions of $c_{2}(\eta), c_{3}(\eta)$ [see Eq. (2.15)], and $\beta \mu^{\mathrm{ex}}(\eta)$ according to several approximations.

\begin{tabular}{cccc}
\hline \hline Approx. & $c_{2}(\eta)$ & $c_{3}(\eta)$ & $\beta \mu^{\mathrm{ex}}(\eta)$ \\
\hline PY-v & $9 \ln (1-\eta)+12 \frac{\eta}{1-\eta}$ & $-6 \ln (1-\eta)-\eta \frac{5-11 \eta}{(1-\eta)^{2}}$ & $2 \ln (1-\eta)+2 \eta \frac{5-2 \eta}{(1-\eta)^{2}}$ \\
PY-c & $3 \eta \frac{2+\eta}{2(1-\eta)^{2}}$ & $\eta \frac{1+\eta+\eta^{2}}{(1-\eta)^{3}}$ & $-\ln (1-\eta)+\eta \frac{14-13 \eta+5 \eta^{2}}{2(1-\eta)^{3}}$ \\
PY- $\mu$ & $27 \frac{\ln (1-\eta)}{\eta}+3 \frac{18-7 \eta}{2(1-\eta)}$ & $-27 \frac{\ln (1-\eta)}{\eta}-\frac{54-83 \eta+14 \eta^{2}}{2(1-\eta)^{2}}$ & $-\ln (1-\eta)+\eta \frac{14+\eta}{2(1-\eta)^{2}}$ \\
BMCSL & $3 \ln (1-\eta)+3 \eta \frac{2-\eta}{(1-\eta)^{2}}$ & $-2 \ln (1-\eta)-\eta \frac{1-6 \eta+3 \eta^{2}}{(1-\eta)^{3}}$ & $\eta \frac{8-9 \eta+3 \eta^{2}}{(1-\eta)^{3}}$ \\
BCSK & $8 \ln (1-\eta)+\eta \frac{22-21 \eta+4 \eta^{2}}{2(1-\eta)^{2}}$ & $-\frac{16}{3} \ln (1-\eta)-\eta \frac{13-43 \eta+27 \eta^{2}-2 \eta^{3}}{3(1-\eta)^{3}}$ & $\frac{5}{3} \ln (1-\eta)+\eta \frac{58-79 \eta+39 \eta^{2}-8 \eta^{3}}{6(1-\eta)^{3}}$ \\
\hline \hline
\end{tabular}

Therefore, since $\lim _{\sigma_{0} \rightarrow \infty} \beta \mu_{0}^{\mathrm{ex}}\left(\eta, \sigma_{0}\right) / \sigma_{0}^{3}$ can be neither zero nor infinity, the only polynomial approximations consistent with that property are third-degree ones.

In the case of the approximations of the form (2.3), Eq. (2.18) implies

$$
c_{3}(\eta)=\eta Z(\eta)=\eta\left[Z_{0}(\eta)+Z_{1}(\eta)+Z_{2}(\eta)\right] .
$$

It can be noticed that Eq. (2.19) is independent of Eq. (2.17). In fact, it can be easily checked that the PY-v, PY- $\mu$, BMCSL, and BCSK expressions for $Z_{2}(\eta)$ (see Table I) and $c_{3}(\eta)$ (see Table II) are inconsistent with Eq. (2.19). This means that those approximations qualitatively agree with the physical requirement (2.18) in that $\lim _{\sigma_{0} \rightarrow \infty} \beta \mu_{0}^{\mathrm{ex}}\left(\eta, \sigma_{0}\right) / \sigma_{0}^{3}=$ finite but yield different results for the left- and right-hand sides. On the other hand, the PY-c approximation, which actually is equivalent to the Scaled Particle Theory (SPT) approximation, ${ }^{31-35}$ is fully consistent with Eqs. (2.18) and (2.19). As a matter of fact, the PY-c/SPT cubic prescription for $\beta \mu_{0}^{\mathrm{ex}}\left(\eta, \sigma_{0}\right)$ is the only one that is simultaneously consistent with both Eqs. (2.17) and (2.19) without violating the value $b_{3}=10$ for the third virial coefficient of the one-component fluid. Combination of Eqs. (2.17) and (2.19) [together with Eqs. (2.9) and (2.16a)] yields the differential equation $a_{2}^{\prime}(\eta)=2 a_{2}(\eta) / \eta(1-\eta)$, whose general solution is $a_{2}(\eta)=K \eta^{2} /(1-\eta)^{2}, K$ being a constant. The associated third virial coefficient is $b_{3}=$ $7+2 K$, so that $b_{3}=10$ implies $K=\frac{3}{2}$ and thus one recovers the $\mathrm{PY}-\mathrm{c} / \mathrm{SPT}$ approximation.

Section III describes the process and results of a MD simulation study of this HS system which were carried out to help determine which of the approximations for $c_{2}$ and $c_{3}$ (see Table II) is best.

\section{WIDOM'S PARTICLE INSERTION METHOD AND MOLECULAR DYNAMICS SIMULATION}

Consider an $N$-particle system where $\Phi_{N}\left(\mathbf{r}^{N}\right)$ is the potential energy. The Widom particle insertion method for the excess chemical potential $\mu^{\text {ex }}$ is $3,6,36,37$

$$
\begin{aligned}
e^{-\beta \mu^{\mathrm{ex}}} & =\frac{\int d \mathbf{r}^{N+1} e^{-\beta \Phi_{N}\left(\mathbf{r}^{N}\right)} e^{-\beta \Delta \Phi_{N+1}\left(\mathbf{r}^{N+1}\right)}}{V \int d \mathbf{r}^{N} e^{-\beta \Phi_{N}\left(\mathbf{r}^{N}\right)}} \\
& =\left\langle e^{-\beta \Delta \Phi_{N+1}\left(\mathbf{r}^{N+1}\right)}\right\rangle
\end{aligned}
$$

where $\Delta \Phi_{N+1}\left(\mathbf{r}^{N+1}\right)=\Phi_{N+1}\left(\mathbf{r}^{N+1}\right)-\Phi_{N}\left(\mathbf{r}^{N}\right)$ and the ensemble average is denoted by $\langle\cdots\rangle$. The $(N+1)$ th particle (here denoted by the subscript 0 ) can be considered to be a test particle, as it does not influence the physical distribution of the other $N$ particles. Hence,

$$
\beta \mu_{0}^{\mathrm{ex}}=-\ln \left\langle e^{-\beta \Delta \Phi_{N+1}\left(\mathbf{r}^{N+1}\right)}\right\rangle .
$$

The test particle is inserted randomly into the $N$ particle host fluid. The important point is that it does so in a non-intrusive way. For HSs, Eq. (3.2) reduces to a simple bookkeeping procedure as $\exp \left(-\beta \Delta \Phi_{N+1}\right)$ either is 1 when the test sphere does not overlap with any of the $N$ particles or is equal to 0 if it overlaps with any of them. As discussed in Sec. II, the test particle does not need to be the same type of particle as the other $N$ particles. We consider particle $\alpha=0$ to be an impurity HS of diameter $\sigma_{0}$, taking the HS diameter of the host fluid to be $\sigma$.

Our numerical implementation of the Widom insertion method run as follows. At a given packing fraction $\eta$, a monodisperse HS fluid was simulated by a standard MD method. The procedure was to randomly insert a test "point" in the system and calculate the distance $r_{n}$ from that point to the center of the nearest sphere. Then, all the values from $\sigma_{0}=0$ to $\sigma_{0}=2 r_{n}-\sigma$ represented accepted insertions, which were accumulated efficiently in a histogram at the same time in the MD simulation. In addition, as the test particles are introduced in a non-intrusive way, many of them can be inserted at the same time, and we used the same number of test particles as the number of host fluid particles. One difference with the LS method ${ }^{9}$ is that we use MD rather than MC to evolve the host fluid assembly of HSs.

For each trial insertion $r_{n}$, 1 was added to all entrants of a histogram (rather like that for the radial 


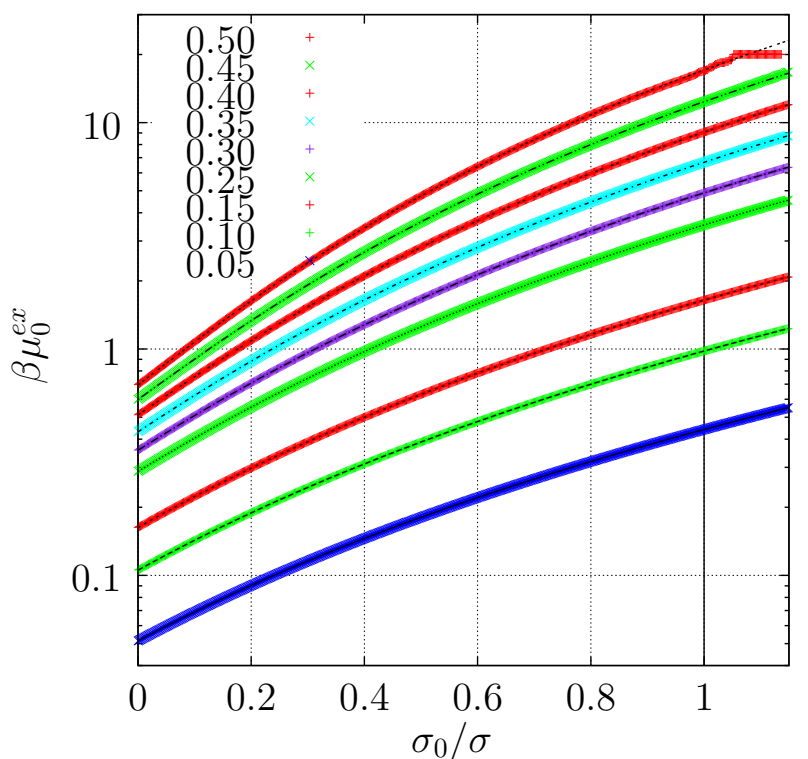

FIG. 1. Plot of the excess chemical potential of a test particle, $\beta \mu_{0}^{\mathrm{ex}}\left(\eta, \sigma_{0}\right)$, as a function of the diameter $\sigma_{0}$. The symbols are the values obtained in our MD simulations by the Widom insertion method, while the lines are least-square fits to cubic polynomials of the form (2.15) with free coefficients $c_{n}$. The noisiest data for large $\eta$ and $\sigma_{0}$ were excluded from the fits. The different values of $\eta$ are indicated in the legend.

distribution function) for $P_{0}\left(\eta, \sigma_{0}\right)$ for $\sigma_{0}=2 r_{n}-\sigma$ and all $\sigma_{0}$ values less than $2 r_{n}-\sigma$ at the same time. This is a statistically efficient procedure for computing the chemical potential of the impurity at infinite dilution, $\beta \mu_{0}^{\mathrm{ex}}\left(\eta, \sigma_{0}\right)$. The chemical potential of the HS fluid is just $\mu^{\mathrm{ex}}(\eta)=\mu_{0}^{\mathrm{ex}}(\eta, \sigma)$ when $\sigma_{0}=\sigma$. At not too high densities, data on the chemical potential for $\sigma_{0}>\sigma$ can also be obtained, and so the HS chemical potential becomes a matter of interpolation and data fitting in that case. For states near a packing fraction $\eta \approx 0.50$ the HS chemical potential needs to be estimated by extrapolation of the $\sigma_{0}<\sigma$ histogram entrants, as the probability of inserting a HS in a HS fluid during a typical simulation can be impracticably small (less than $\left.10^{-7}\right)$.

At each density, the MD values of $\beta \mu_{0}\left(\eta, \sigma_{0}\right)$ as a function of $\sigma_{0}$ were fitted to the cubic polynomial (2.15) to obtain the four coefficients $c_{0}-c_{3}$, without imposing the exact values $(2.9 \mathrm{a})$ and $(2.9 \mathrm{~b})$ of $c_{0}$ and $c_{1}$. This contrasts with the LS procedure, ${ }^{9}$ where the coefficients $c_{0}$ and $c_{1}$ were fixed to be given by Eqs. (2.9a) and (2.9b), the coefficient $c_{3}$ was forced to satisfy the relationship (2.17) (with $Z$ obtained by independent MC simulations of the host fluid), and therefore only the coefficient $c_{2}$ was fitted to the simulation data of $-\ln P_{0}\left(\eta, \sigma_{0}\right)$. In addition, the maximum value of $\sigma_{0}$ used in the least-square fitting corresponded to ${ }^{9} P_{0} \approx 10^{-3}$.

Our simulations were carried out with $N=2048$ HSs. There were ca. $1.4 \times 10^{5}$ collisions per particle at $\eta=0.05$

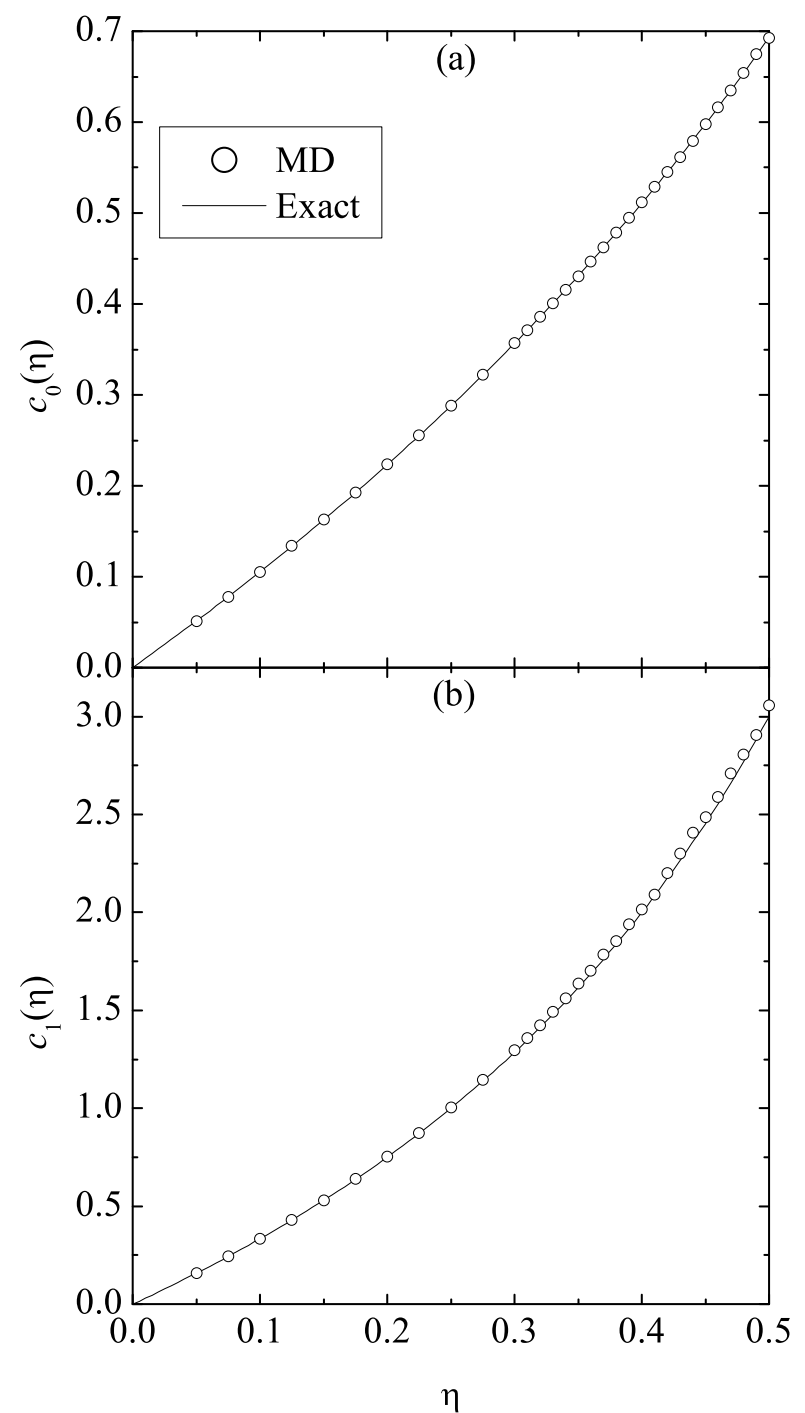

FIG. 2. Plot of the coefficients (a) $c_{0}(\eta)$ and (b) $c_{1}(\eta)$. The lines represent the exact expressions [see Eqs. (2.9a) and $(2.9 \mathrm{~b})]$, while the symbols represent the values obtained from a least-square fit of MD data.

and $5.6 \times 10^{5}$ collisions per particle at $\eta=0.5$. The maximum value of $\sigma_{0}$ chosen for the fitting process was $1.10 \sigma$, for $\eta<0.4$, decreasing to $0.90 \sigma$ for $\eta=0.46$ to $0.80 \sigma$ for $\eta \geq 0.48$. This corresponded to $P_{0} \approx 2 \times 10^{-5}$. The insertion probability histogram had a resolution of $0.005 \sigma$.

\section{RESULTS}

Figure 1 shows the values of $\beta \mu_{0}^{\mathrm{ex}}\left(\eta, \sigma_{0}\right)$ obtained in our simulations for nine representative packing fractions from $\eta=0.05$ to $\eta=0.50$. The least-square fits to a cubic polynomial are also included in Fig. 1 and an excellent agreement is found.

The extracted values of the coefficients $c_{0}(\eta)$ and $c_{1}(\eta)$ 


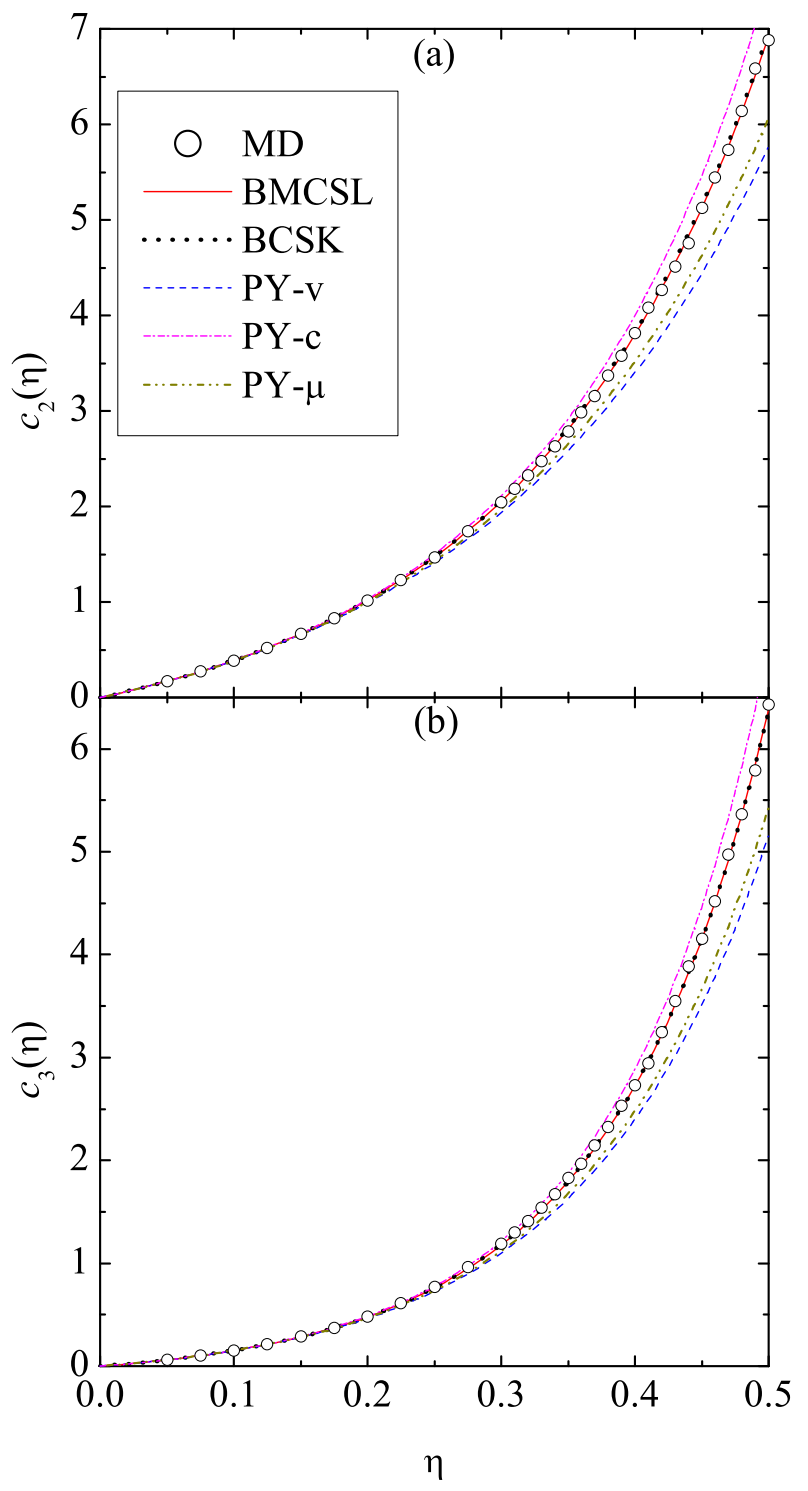

FIG. 3. Plot of the coefficients (a) $c_{2}(\eta)$ and (b) $c_{3}(\eta)$. The lines represent the theoretical expressions (see Table II), while the symbols represent the values obtained from a least-square fit of MD data.

are plotted in Fig. 2 for 31 values of $\eta$ ranging from 0.05 to 0.50 . Comparison with the exact expressions (2.9a) and $(2.9 \mathrm{~b})$ shows an extremely good agreement. This confirms and reinforces the reliability and accuracy of our MD results.

Figure 3 displays the values of the fitted coefficients $c_{2}(\eta)$ and $c_{3}(\eta)$ for the same densities as in Fig. 2. Since the exact expressions of $c_{2}$ and $c_{3}$ are (to the best of our knowledge) unknown, we compare the simulation values with the approximate theoretical predictions considered in Table II. Up to $\eta \simeq 0.2$ all the theories practically overlap and reproduce the MD values. At higher densities, however, the three PY predictions clearly deviate from the simulation data: while the PY-c approximation overestimates the data, the PY- $\mu$ and, especially, the PY-v

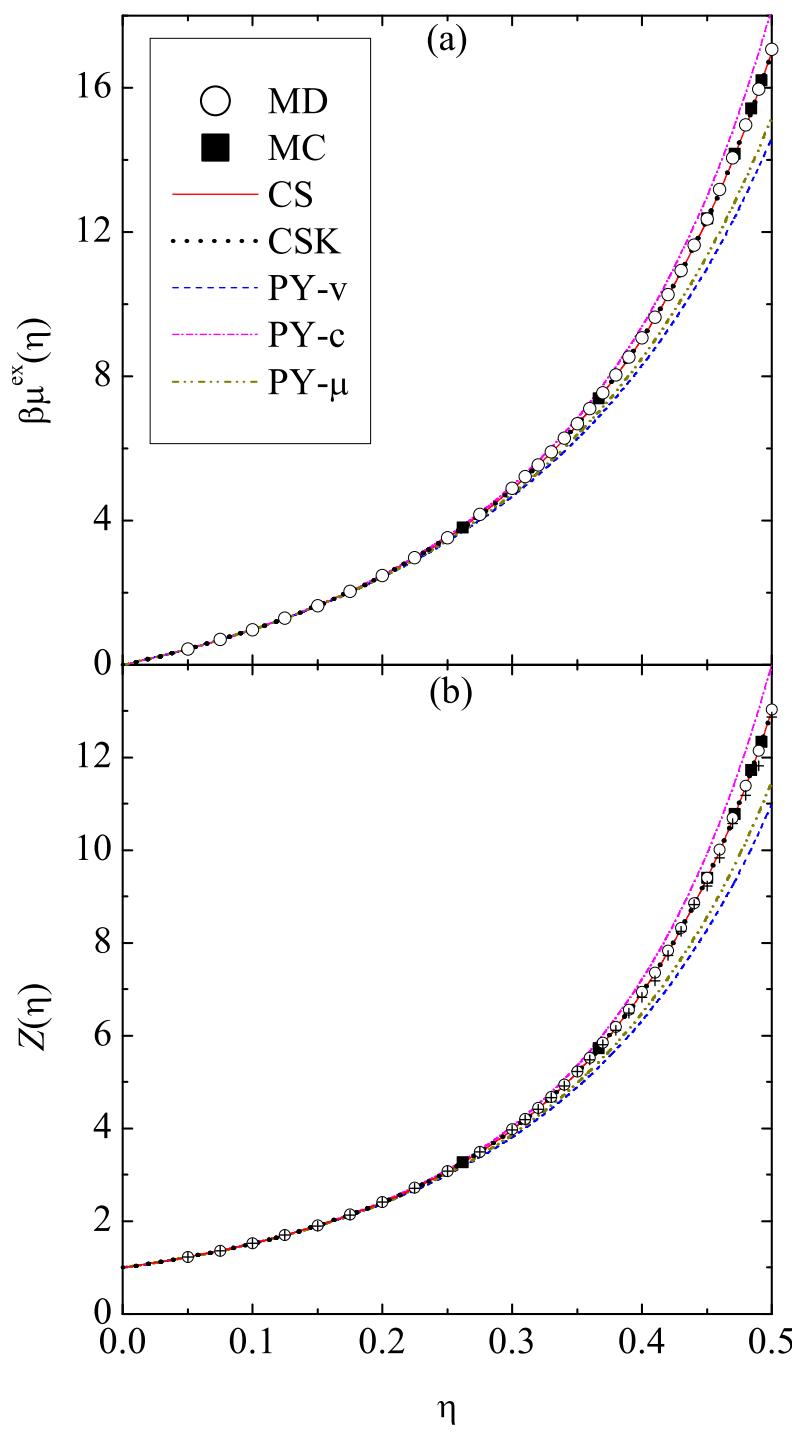

FIG. 4. Plot of (a) the excess chemical potential $\beta \mu^{\mathrm{ex}}(\eta)$ and (b) the compressibility factor $Z(\eta)$. The lines represent the theoretical expressions (see Tables I and II), the open circles represent $\beta \mu^{\mathrm{ex}}=c_{0}+c_{1}+c_{2}+c_{3}$ and $Z=1+\frac{1}{3} c_{1}+\frac{2}{3} c_{2}+c_{3}$ (with coefficients $c_{n}$ obtained from a least-square fit of our MD data), and the filled squares represent the MC data of Ref. 9. In panel (b), the crosses represent $c_{3} / \eta$.

approximations underestimate them. On the other hand, the BMCSL and BCSK curves, which are practically indistinguishable, reproduce excellently the MD results.

Now that we have validated our numerical values of the four coefficients $c_{n}$ characterizing the diameter dependence of the impurity chemical potential $\beta \mu_{0}^{\mathrm{ex}}$, an accurate estimate of the chemical potential of the pure HS fluid, written as $\beta \mu^{\mathrm{ex}}=c_{0}+c_{1}+c_{2}+c_{3}$, can be made. The results are shown in Fig. 4(a), where they are compared with the PY, CS, and CSK approximations (see again Table II). The observed trends are similar to those presented in Fig. 3. In particular, there is excellent agreement between the present MD results and the 
TABLE III. Numerical values of $c_{0}, c_{1}, c_{2}, c_{3}, \beta \mu^{\mathrm{ex}}=c_{0}+c_{1}+c_{2}+c_{3}$, and $Z=1+\frac{1}{3} c_{1}+\frac{2}{3} c_{2}+c_{3}$, as obtained from our MD simulations. The errors in the constants $c_{0}-c_{3}$ range from $0.002-0.01 \%$ at $\eta=0.075$ to $0.06-0.15 \%$ at $\eta=0.50$.

\begin{tabular}{|c|c|c|c|c|c|c|}
\hline$\eta$ & $c_{0}$ & $c_{1}$ & $c_{2}$ & $c_{3}$ & $\beta \mu^{\mathrm{ex}}$ & $Z$ \\
\hline 0.050 & 0.05138 & 0.15791 & 0.17053 & 0.06132 & 0.4411 & $\overline{1.2276}$ \\
\hline 0.075 & 0.07807 & 0.24363 & 0.27251 & 0.10232 & 0.6965 & 1.3652 \\
\hline 0.100 & 0.10551 & 0.33413 & 0.38726 & 0.15254 & 0.9794 & 1.5221 \\
\hline 0.125 & 0.13371 & 0.42976 & 0.51731 & 0.21300 & 1.2938 & 1.7011 \\
\hline 0.150 & 0.16277 & 0.53066 & 0.66523 & 0.28545 & 1.6441 & 1.9058 \\
\hline 0.175 & 0.19264 & 0.63853 & 0.83044 & 0.37440 & 2.0360 & 2.1409 \\
\hline 0.200 & 0.22340 & 0.75338 & 1.01629 & 0.48254 & 2.4756 & 2.4112 \\
\hline 0.225 & 0.25519 & 0.87520 & 1.22927 & 0.61128 & 2.9709 & 2.7225 \\
\hline 0.250 & 0.28802 & 1.00569 & 1.46854 & 0.76883 & 3.5311 & 3.0831 \\
\hline 0.275 & 0.32190 & 1.14594 & 1.73817 & 0.96082 & 4.1668 & 3.5016 \\
\hline 0.300 & 0.35694 & 1.29667 & 2.04555 & 1.19320 & 4.8924 & 3.9891 \\
\hline 0.310 & 0.37134 & 1.35932 & 2.18212 & 1.29733 & 5.2101 & 4.2052 \\
\hline 0.320 & 0.38594 & 1.42389 & 2.32556 & 1.41204 & 5.5474 & 4.4370 \\
\hline 0.330 & 0.40065 & 1.49284 & 2.47122 & 1.53868 & 5.9034 & 4.6838 \\
\hline 0.340 & 0.41582 & 1.56042 & 2.63206 & 1.67237 & 6.2807 & 4.9472 \\
\hline 0.350 & 0.43065 & 1.63751 & 2.78336 & 1.82938 & 6.6809 & 5.2308 \\
\hline 0.360 & 0.44664 & 1.70377 & 2.98153 & 1.97059 & 7.1025 & 5.5262 \\
\hline 0.370 & 0.46210 & 1.78329 & 3.15755 & 2.14789 & 7.5508 & 5.8473 \\
\hline 0.380 & 0.47863 & 1.85519 & 3.37150 & 2.32390 & 8.0292 & 6.1900 \\
\hline 0.390 & 0.49464 & 1.93913 & 3.57437 & 2.52817 & 8.5363 & 6.5575 \\
\hline 0.400 & 0.51157 & 2.01626 & 3.81678 & 2.72871 & 9.0733 & 6.9453 \\
\hline 0.410 & 0.52923 & 2.09101 & 4.08354 & 2.94310 & 9.6469 & 7.3625 \\
\hline 0.420 & 0.54483 & 2.20251 & 4.26902 & 3.24896 & 10.265 & 7.8291 \\
\hline 0.430 & 0.56175 & 2.30181 & 4.51157 & 3.54899 & 10.924 & 8.3240 \\
\hline 0.440 & 0.57897 & 2.40785 & 4.75777 & 3.88409 & 11.629 & 8.8586 \\
\hline 0.450 & 0.59808 & 2.48743 & 5.12705 & 4.15586 & 12.368 & 9.4030 \\
\hline 0.460 & 0.61655 & 2.58968 & 5.44313 & 4.52232 & 13.172 & 10.014 \\
\hline 0.470 & 0.63462 & 2.71022 & 5.73096 & 4.97255 & 14.048 & 10.697 \\
\hline 0.480 & 0.65443 & 2.80728 & 6.14140 & 5.36583 & 14.969 & 11.396 \\
\hline 0.490 & 0.67473 & 2.90778 & 6.58217 & 5.79039 & 15.955 & 12.148 \\
\hline 0.500 & 0.69285 & 3.05778 & 6.87840 & 6.43386 & 17.063 & 13.039 \\
\hline
\end{tabular}

CS and CSK theories. Figure 4(a) also includes the MC data reported in Ref. 9, which are fully consistent with our MD results.

An interesting additional feature of our approach is that we can predict the compressibility factor $Z(\eta)$ of the HS fluid via Eq. (2.17) from the knowledge of the coefficients $c_{n}$ characterizing the size dependence of the solute chemical potential $\beta \mu_{0}^{\mathrm{ex}}$, i.e., $Z=1+\frac{1}{3} c_{1}+\frac{2}{3} c_{2}+c_{3}$. This quantity is plotted in Fig. 4(b), where it shows again an excellent agreement with the CS and CSK approximations, as well as with the results obtained in Ref. 9 directly from MC simulations of the radial distribution function at contact.

In principle, one could also estimate $Z$ only from $c_{3}$ as $Z=c_{3} / \eta$ [see Eq. (2.19)]. As shown in Fig. 4(b), the values of $c_{3} / \eta$ agree very well with those of $1+\frac{1}{3} c_{1}+\frac{2}{3} c_{2}+c_{3}$ up to $\eta \simeq 0.35$, but tend to lie slightly below the latter ones at higher densities. This small discrepancy is just a consequence of the fact that the exact function $\beta \mu_{0}^{\mathrm{ex}}\left(\eta, \sigma_{0}\right)$ is not a cubic polynomial. In fact, as discussed at the end of Sec. II, the only cubic polynomial that is consistent with both Eqs. (2.17) and (2.19) is the PY-c/SPT approximation, which is not particularly accurate. Our MD results show that the excess chemical potential $\beta \mu_{0}^{\mathrm{ex}}\left(\eta, \sigma_{0}\right)$ can be fitted extremely well by a cubic polynomial for diameters $\sigma_{0}$ from $\sigma_{0}=0$ to $\sigma_{0} \sim \sigma$ (see Fig. 1). On the other hand, while the choice of the degree of the polynomial is consistent with the exact property $\lim _{\sigma_{0} \rightarrow \infty} \beta \mu_{0}^{\mathrm{ex}}\left(\eta, \sigma_{0}\right) /\left(\sigma_{0} / \sigma\right)^{3}=$ finite, it would be too far-fetched to expect that such an extreme limit coincides with the coefficient $c_{3}$ fitted in the range $0 \leq \sigma_{0} \lesssim \sigma$. The fact, however, that the coefficient $c_{3}$ is so close to $\eta Z$ means that the cubic polynomial fitted in the range $0 \leq \sigma_{0} \lesssim \sigma$ keeps being a very good approximation even if $\sigma_{0} \gg \sigma$. Anyway, the recommended route to measure the compressibility factor from a fit of the form (2.15) for $\sigma_{0} \lesssim \sigma$ is $Z=1+\frac{1}{3} c_{1}+\frac{2}{3} c_{2}+c_{3}$ rather than $Z=c_{3} / \eta$.

For future reference of researchers interested in the chemical potential of HS fluids, we present in Table III the numerical values of the MD data plotted in Figs. 2-4.

\section{CONCLUSIONS}

To conclude, this work provides new insights into the properties of the chemical potential of HS fluids and its relation with the equation of state. A third-degree expression in the test particle diameter for the chemical potential is shown to reproduce well that for HSs with the same diameter as those of the host fluid, and also for those tracer particles with smaller and, to some extent, larger diameters (not too close to $\eta=0.49$ in the latter case). It is found that the chemical potential predicted by the the CS and the CSK equations is in close agreement with simulation data. However, it is concluded that although a third-degree polynomial in tracer particle diameter is a very good approximation of the chemical potential, this functional form cannot be exact. It is also shown that the equation of state of the HS fluid can be 
accurately obtained from the polynomial fit of the simulation data for the chemical potential.

Originally implemented on NVT MC simulations, we have applied in this paper the LS technique ${ }^{9}$ to MD simulations. In addition, our implementation differs from that of Ref. 9 in a few aspects. First, all four coefficients $c_{0}-c_{3}$ have been fitted, whereas LS forced $c_{0}$ and $c_{1}$ to be equal to their exact values and enslaved $c_{3}$ to $c_{2}$ by means of Eq. (2.17), so that in the end only the coefficient $c_{2}$ was fitted. Also, they needed to measure the compressibility factor $Z$ (from the contact value of the radial distribution function) independently of the insertion probability measurements, whereas in our case $Z$ is just another output (in addition to $\beta \mu^{\mathrm{ex}}$ ) rather than an input. The excellent agreement between the fitted $c_{0}$ and $c_{1}$ with the exact expressions is an a posteriori confirmation of the accuracy of the results reported in this paper. We have been able to reach reliable statistical results up to $P_{0} \approx 2 \times 10^{-5}$, which is about 50 times smaller than the threshold value considered in Ref. 9. Furthermore, our study covers a much larger number of densities.

The LS simulation technique is shown to be an extremely powerful and adaptable tool to obtain the chemical potential of tracer particles and the equation of state of HS fluids. It has also been shown that the BMCSL and BCSK formulas for $c_{2}$ and $c_{3}$ are extremely accurate, and not distinguishable from the numerical data. Therefore it may be concluded that the equation of state of the monodisperse HS fluid can be considered for most practical applications to be a solved analytic problem.

In the extension to HS binary mixtures of the LS method carried out by Barošová et al. ${ }^{11}$ the authors fitted their MC simulated values of $P_{0}\left(\eta, \sigma_{0}\right)$ to a quartic polynomial. On the other hand, we plan to extend our MD implementation to HS mixtures (binary, ternary, or, more generally, polydisperse) by keeping instead a cubic form since the exact condition $\lim _{\sigma_{0} \rightarrow \infty} \beta \mu_{0}^{\mathrm{ex}}\left(\eta, \sigma_{0}\right) / \sigma_{0}^{3}=$ finite still holds for mixtures. According to Eq. (2.12), the coefficient $c_{0}$ is the same as in the monodisperse system, while the linear coefficient, once multiplied by $M_{3} / M_{1} M_{2}$, is again the exact $c_{1}$. As carried out in the present paper, these two conditions will be used as confidence tests of the simulation results.

\section{ACKNOWLEDGMENTS}

The research of A.S. has been partially supported by the Spanish Government through Grant No. FIS201342840-P and by the Regional Government of Extremadura (Spain) through Grant No. GR15104 (par- tially financed by ERDF funds). D.M.H. would like to thank Dr. T. Crane (Department of Physics, Royal Holloway, University of London, UK) for helpful software support.

\section{Appendix A: Chemical potential in the small-size limit}

We consider an $N$-particle HS mixture in $d$ dimensions. The packing fraction of the mixture is $\eta=(N / V) v_{d} M_{d}$, where $v_{d}=(\pi / 4)^{d / 2} / \Gamma(1+d / 2)$ is the volume occupied by a sphere of unit diameter. The Boltzmann factor associated with the potential energy $\Phi_{N}\left(\mathbf{r}^{N}\right)$ of the mixture is

$$
e^{-\beta \Phi_{N}\left(\mathbf{r}^{N}\right)}=\prod_{\alpha=1}^{N-1} \prod_{\gamma=\alpha+1}^{N} \Theta\left(r_{\alpha \gamma}-\sigma_{\ell_{\alpha} \ell_{\gamma}}\right),
$$

where $\Theta(x)$ is the Heaviside step function, $r_{\alpha \gamma}=\mid \mathbf{r}_{\alpha}-$ $\mathbf{r}_{\gamma} \mid$ is the relative distance between particles $\alpha$ and $\gamma$, $\ell_{\alpha}$ denotes the species particle $\alpha$ belongs to, and $\sigma_{i j}=$ $\frac{1}{2}\left(\sigma_{i}+\sigma_{j}\right)$.

Now we assume that an extra test particle of diameter $\sigma_{0}$ is inserted into the fluid. The canonical ensemble expression for the insertion probability is [see Eq. (3.1)]

$$
\begin{aligned}
P_{0}\left(\eta, \sigma_{0}\right) & =\left\langle\prod_{\gamma=1}^{N} \Theta\left(r_{0 \gamma}-\sigma_{0 \ell_{\gamma}}\right)\right\rangle \\
& =\frac{\int d \mathbf{r}^{N} e^{-\beta \Phi_{N}\left(\mathbf{r}^{N}\right)} \int d \mathbf{r}_{0} \prod_{\gamma=1}^{N} \Theta\left(r_{0 \gamma}-\sigma_{0 \ell_{\gamma}}\right)}{V \int d \mathbf{r}^{N} e^{-\beta \Phi_{N}\left(\mathbf{r}^{N}\right)}} .
\end{aligned}
$$

In the limit $\sigma_{0} \rightarrow 0$, we can write

$$
P_{0}\left(\eta, \sigma_{0}\right)=P_{0}(\eta, 0)+\dot{P}_{0}(\eta, 0) \sigma_{0}+\mathcal{O}\left(\sigma_{0}^{2}\right),
$$

where the dot denotes a derivative with respect to $\sigma_{0}$. The first term on the right-hand side of Eq. (A3) is trivial since

$$
\int d \mathbf{r}_{0} \prod_{\gamma=1}^{N} \Theta\left(r_{0 \gamma}-\frac{\sigma_{\ell_{\gamma}}}{2}\right)=V(1-\eta)
$$

This expresses the fact that, for any nonoverlapping configuration of $N$ spheres, the available volume for the test point particle is $V(1-\eta)$. Consequently,

$$
P_{0}(\eta, 0)=1-\eta
$$

As for the derivative $\dot{P}_{0}\left(\eta, \sigma_{0}\right)$, it is given from Eq. (A2) by

$$
\dot{P}_{0}\left(\eta, \sigma_{0}\right)=-\frac{1}{2} \sum_{\alpha=1}^{N} \frac{\int d \mathbf{r}^{N} e^{-\Phi_{N}\left(\mathbf{r}^{N}\right)} \int d \mathbf{r}_{0} \delta\left(r_{0 \alpha}-\sigma_{0 \ell_{\alpha}}\right) \prod_{\gamma \neq \alpha} \Theta\left(r_{0 \gamma}-\sigma_{0 \ell_{\gamma}}\right)}{V \int d \mathbf{r}^{N} e^{-\beta \Phi_{N}\left(\mathbf{r}^{N}\right)}}
$$


Making $\sigma_{0} \rightarrow 0$ and assuming again a nonoverlapping configuration of the fluid particles, we can write

$$
\int d \mathbf{r}_{0} \delta\left(r_{0 \alpha}-\frac{\sigma_{\ell_{\alpha}}}{2}\right) \prod_{\gamma \neq \alpha} \Theta\left(r_{0 \gamma}-\frac{\sigma_{\ell_{\gamma}}}{2}\right)=\Omega_{d} \lim _{\epsilon \rightarrow 0} \int_{0}^{\frac{\sigma_{\ell_{\alpha}}}{2}+\epsilon} d r_{0 \alpha} r_{0 \alpha}^{d-1} \delta\left(r_{0 \alpha}-\frac{\sigma_{\ell_{\alpha}}}{2}\right)=\Omega_{d} 2^{1-d} \sigma_{\ell_{\alpha}}^{d-1},
$$

where $\Omega_{d}=d v_{d} 2^{d}$ is the total solid angle. Therefore,

$$
\dot{P}_{0}(\eta, 0)=-d \eta \frac{M_{d-1}}{M_{d}} .
$$

After insertion of Eqs. (A5) and (A8), Eq. (A3) becomes

$$
P_{0}\left(\eta, \sigma_{0}\right)=(1-\eta)\left(1-d \frac{\eta}{1-\eta} \frac{M_{1} M_{d-1}}{M_{d}} \frac{\sigma_{0}}{M_{1}}\right)+\mathcal{O}\left(\sigma_{0}^{2}\right)
$$

Finally, from Eq. (1.1) we find

$$
\beta \mu_{0}^{\mathrm{ex}}\left(\eta, \sigma_{0}\right)=c_{0}(\eta)+c_{1}(\eta) \frac{M_{1} M_{d-1}}{M_{d}} \frac{\sigma_{0}}{M_{1}}+\mathcal{O}\left(\sigma_{0}^{2}\right)
$$

with

$$
c_{0}(\eta)=-\ln (1-\eta), \quad c_{1}(\eta)=d \frac{\eta}{1-\eta} .
$$

Identifying the test particle as a particle of species $i$ (i.e., $\sigma_{0}=\sigma_{i}$ ) and focusing on $d=3$, it can be readily shown that Eqs. (A10) and (A11) reduce to Eq. (2.14) and $(2.9 \mathrm{a})-(2.9 \mathrm{~b})$, respectively.

Equation (A8) can be obtained by a different route. Imagine a test particle that can (partially) "penetrate" inside the fluid particles, i.e., it has a nominal diameter $\sigma_{0}<0$ so that the closest distance $\sigma_{0 j}$ between the centers of the test particle and a particle of species $j$ is smaller than $\frac{1}{2} \sigma_{j}$. In that case, Eq. (A2) still holds and, in analogy to Eq. (A4),

$$
\int d \mathbf{r}_{0} \prod_{\gamma=1}^{N} \Theta\left(r_{0 \gamma}-\sigma_{0 \ell_{\gamma}}\right)=V-\sum_{j} N_{j} v_{d}\left(2 \sigma_{0 j}\right)^{d} .
$$

Therefore,

$$
\begin{aligned}
& P_{0}\left(\eta, \sigma_{0}<0\right)=1-\frac{1}{V} \sum_{j} N_{j} v_{d}\left(\sigma_{0}+\sigma_{j}\right)^{d}, \\
& \dot{P}_{0}\left(\eta, \sigma_{0}<0\right)=-\frac{d}{V} \sum_{j} N_{j} v_{d}\left(\sigma_{0}+\sigma_{j}\right)^{d-1} .
\end{aligned}
$$

Taking the limit $\sigma_{0} \rightarrow 0$, Eqs. (A13) reduce to Eqs. (A5) and (A8). This in turn shows that both $P_{0}\left(\eta, \sigma_{0}\right)$ and $\dot{P}_{0}\left(\eta, \sigma_{0}\right)$ are continuous at $\sigma_{0}=0$.
${ }^{1}$ A. Mulero, ed., Theory and Simulation of Hard-Sphere Fluids and Related Systems, vol. 753 of Lecture Notes in Physics (Springer-Verlag, Berlin, 2008).

${ }^{2}$ V. Baranau and U. Tallarek, J. Chem. Phys. 144, 214503 (2016).

${ }^{3}$ B. Widom, J. Chem. Phys. 39, 2808 (1963).

${ }^{4}$ K. S. Shing and K. E. Gubbins, Mol. Phys. 43, 717 (1981).

${ }^{5}$ G. L. Deitrick, L. E. Scriven, and H. T. Davis, J. Chem. Phys. 90, 2370 (1989).

${ }^{6}$ W. G. Hoover and J. C. Poirier, J. Chem. Phys. 37, 1041 (1962).

${ }^{7}$ I. Nezbeda and J. Kolafa, Mol. Simul. 5, 391 (1991).

${ }^{8}$ P. Attard, J. Chem. Phys. 98, 2225 (1993).

${ }^{9}$ S. Labík and W. R. Smith, Mol. Simul. 12, 23 (1994).

${ }^{10}$ S. Labík, V. Jirásek, A. Malijevský, and W. Smith, Chem. Phys. Lett. 247, 227 (1995).

${ }^{11}$ M. Barošová, A. Malijevský, S. Labík, and W. R. Smith, Mol. Phys. 87, 423 (1996).

${ }^{12}$ A. Mulero, C. A. Galán, M. I. Parra, and F. Cuadros, in Theory and Simulation of Hard-Sphere Fluids and Related Systems, edited by A. Mulero (Springer-Verlag, Berlin, 2008), vol. 753 of Lecture Notes in Physics, pp. 37-109.

${ }^{13}$ C. Barrio and J. R. Solana, in Theory and Simulation of Hard-Sphere Fluids and Related Systems, edited by A. Mulero (Springer-Verlag, Berlin, 2008), vol. 753 of Lecture Notes in Physics, pp. 133-182.

${ }^{14}$ J. L. Lebowitz and D. Zomick, J. Chem. Phys. 54, 3335 (1971).

15 J. W. Perram and E. R. Smith, Chem. Phys. Lett. 35, 138 (1975).

${ }^{16}$ B. Barboy, Chem. Phys. 11, 357 (1975).

${ }^{17}$ J. K. Percus and G. J. Yevick, Phys. Rev. 110, 1 (1958).

${ }^{18}$ A. Santos, A Concise Course on the Theory of Classical Liquids. Basics and Selected Topics, vol. 923 of Lecture Notes in Physics (Springer, New York, 2016).

${ }^{19}$ A. Santos, Phys. Rev. Lett. 109, 120601 (2012).

${ }^{20}$ A. Santos and R. D. Rohrmann, Phys. Rev. E 87, 052138 (2013).

${ }^{21}$ T. Boublík, J. Chem. Phys. 53, 471 (1970).

${ }^{22}$ G. A. Mansoori, N. F. Carnahan, K. E. Starling, and J. T. W. Leland, J. Chem. Phys. 54, 1523 (1971).

${ }^{23}$ N. F. Carnahan and K. E. Starling, J. Chem. Phys. 51, 635 (1969).

${ }^{24}$ D. M. Heyes, M. J. Cass, J. G. Powles, and W. A. B. Evans, J. Phys. Chem. B 111, 1455 (2007).

${ }^{25}$ T. Boublík and I. Nezbeda, Coll. Czech. Chem. Commun. 51, 2301 (1986).

${ }^{26} \mathrm{~J}$. Kolafa, private communication (1998).

${ }^{27}$ T. Boublík, Mol. Phys. 59, 371 (1986).

${ }^{28}$ H. Reiss, H. L. Frisch, E. Helfand, and J. L. Lebowitz, J. Chem. Phys. 32, 119 (1960).

${ }^{29}$ R. Roth, R. Evans, A. Lang, and G. Kahl, J. Phys.: Condens. Matter 14, 12063 (2002).

${ }^{30}$ A. Santos, Phys. Rev. E 86, 040102(R) (2012).

${ }^{31}$ H. Reiss, H. L. Frisch, and J. L. Lebowitz, J. Chem. Phys. 31, 369 (1959).

32 J. L. Lebowitz, E. Helfand, and E. Praestgaard, J. Chem. Phys. 43, 774 (1965).

${ }^{33}$ M. Mandell and H. Reiss, J. Stat. Phys. 13, 113 (1975).

${ }^{34}$ Y. Rosenfeld, J. Chem. Phys. 89, 4272 (1988).

${ }^{35}$ M. Heying and D. S. Corti, Fluid Phase Equil. 220, 85 (2004).

${ }^{36}$ K.-K. Han, J. H. Cushman, and D. J. Diestler, J. Chem. Phys. 93, 5167 (1990).

${ }^{37}$ D. M. Heyes, Chem. Phys. 159, 149 (1992). 